\title{
The Utility of Molecular Imaging in Prostate Cancer
}

\author{
Aaron Leiblich $^{1} \cdot$ Daniel Stevens $^{2} \cdot$ Prasanna Sooriakumaran $^{3}$
}

Published online: 19 February 2016

(C) The Author(s) 2016. This article is published with open access at Springerlink.com

\begin{abstract}
Prostate cancer is the commonest solid-organ cancer diagnosed in males and represents an important source of morbidity and mortality worldwide. Imaging plays a crucial role in diagnosing prostate cancer and informs the ongoing management of the disease at all stages. Several novel molecular imaging technologies have been developed recently that have the potential to revolutionise disease diagnosis and the surveillance of patients living with prostate cancer. These innovations include hyperpolarised MRI, choline PET/CT and PSMA PET/CT. The major utility of choline and PSMA PET/ CT currently lies in their sensitivity for detecting early recurrence after radical treatment for prostate cancer and identifying discrete lesions that may be amenable to salvage therapy. Molecular imaging is likely to play a future role in characterising genetic and biochemical signatures in individual tumours, which may be of particular significance as cancer therapies move into an era of precision medicine.
\end{abstract}

This article is part of the Topical Collection on Urosurgery

Aaron Leiblich

aleiblich@gmail.com

Daniel Stevens

Daniel.stevens@linacre.ox.ac.uk

Prasanna Sooriakumaran

prasanna.sooriakumaran@nds.ox.ac.uk

1 Department of Urology, Churchill Hospital, University of Oxford NHS Trust, Old Road, Headington, Oxford, UK

2 Department of Physiology, Anatomy and Genetics, University of Oxford, Le Gros Clark Building, South Parks Road, Oxford OX1 3QX, UK

3 Nuffield Department of Surgical Sciences, University of Oxford, John Radcliffe Hospital, Oxford OX3 9DU, UK
Keywords Surgery $\cdot$ Prostate cancer $\cdot$ MRI $\cdot$ PET/CT $\cdot$ PSMA

\section{Introduction}

Prostate cancer is the most frequently diagnosed malignancy in men and is the second commonest cause of male cancerrelated deaths in Europe and North America [1,2]. Disease that is organ-confined at the time of diagnosis is associated with a good prognosis and is frequently amenable to curative therapy $[2,3]$. Conversely, metastatic disease heralds a poor prognosis; men with skeletal metastases have a 5-year survival rate below $30 \%[4,5]$.

Molecular imaging describes a discipline that emerged in the early 2000s and facilitates the visualisation of biological interactions at the cellular level [6]. Unlike traditional imaging modalities, such as ultrasound or conventional CT, molecular imaging techniques are able to probe the biochemical abnormalities that underpin a particular disease process. Clearly, this will prove an invaluable tool for planning individualised treatments as we progress toward an era of personalised medicine, whereby cancer is viewed as a genetically and biochemically heterogeneous disease best managed with tailored therapies [7-10].

This review aims to discuss molecular imaging techniques currently employed in the assessment of prostate cancer and to also familiarise the reader with ongoing developments that are likely to have clinical application in the near future.

\section{Molecular Imaging as a Diagnostic and Staging Tool in Newly Diagnosed Prostate Cancer}

Radiological imaging plays an important role in the diagnosis and initial staging of prostate cancer. For many decades, trans- 
rectal ultrasound (TRUS)-guided biopsy has served as the standard technique for obtaining prostatic tissue for diagnosis. In recent years, multi-parametric MRI (MP-MRI) has emerged as a vital tool for the diagnosis and accurate staging of prostate cancer [11, 12]. To date, no CT- and/or PET-based imaging modalities have demonstrated any benefit over MRI in the imaging of early prostate cancer [13-15]. However, advances in MR spectroscopy seem likely to permit technologies for use in clinical contexts that provide data on tumour metabolism, thus helping to establish the aggressiveness of discrete malignant foci.

A promising development in this area is the emergence of hyperpolarised $\left[1-{ }^{13} \mathrm{C}\right]$ pyruvate as an adjunct to conventional MR. Hyperpolarised MR is a novel molecular imaging technique that can be used to monitor the metabolism of endogenous biomolecules $[16 \cdot, 17]$. Pre-clinical mouse models of prostate cancer have described increased signal detected by MR from hyperpolarised lactate in malignant relative to normal tissues, with levels of both hyperpolarised lactate and pyruvate seen to increase with progression and reduce after therapy $[18,19]$. A promising study in humans demonstrates the safety and feasibility of hyperpolarised pyruvate injections in men with prostate cancer [16•]. This study demonstrates that elevated levels of hyperpolarised pyruvate are observed in regions of biopsy-proven prostate cancer. In addition to its use as a diagnostic tool, it seems probable that refinements in this approach will provide insights into individual tumour biology and behaviour. If this comes to fruition, hyperpolarised MR will undoubtedly aid the risk stratification of organconfined malignancy at the time of diagnosis and also serve as a powerful tool to monitor disease in patients managed on an active surveillance protocol.

\section{Detecting Early Recurrence After Radical Therapy}

The aim of radical treatment in prostate cancer is to cure men of their disease. Unfortunately, a proportion of men will develop disease relapse despite radical therapy. Therefore, an important aspect of the management of men who have undergone radical therapy involves surveillance and detection of recurrence. A rising PSA level after radical therapy is indicative of disease relapse. The management of recurrent disease will depend on several factors, including the PSA velocity and extent and location of disease [20]. Advances in molecular imaging provide new opportunities to assess the extent and distribution of recurrent disease. Choline PET/CT is a technique that is becoming increasingly employed as a standard imaging modality in cases of suspected relapse. More recently, the imaging agent $\mathrm{Ga}-68$ prostate-specific membrane antigen (Ga-68 PSMA) used in conjunction with PET/CT has been shown to detect early recurrence with very high sensitivity [21].

\section{Choline PET Scanning as a Tool for Identifying Early Recurrence}

A study from 2011 evaluated 43 patients who underwent radical prostatectomy with 31 days after C11-choline PET scan [22]. Trans-axial images and histological specimens were analysed by comparing the respective slices. This study demonstrated that small tumours $(<5 \mathrm{~mm})$ were poorly detected while larger tumours tended to be well delineated by PET.

The greatest utility of choline PET scanning appears to be in the context of rising PSA following definitive local therapy. This is of particular use in patients with biochemical relapse, whereby PSA elevation is the only manifestation of their disease. In this context, choline PET scanning can help distinguish between localised, regional and distant disease recurrence. This informs disease management as the extent of recurrence helps define treatment options. For example, small areas of local recurrence may be amenable to salvage radiotherapy, whereas early detection of distant disease may facilitate the timely introduction of anti-androgen therapy (ADT) [23]. In one study of 63 patients with biochemical relapse, $56 \%$ (35/ 63) had abnormal choline PET scans. Recurrent disease could be localised in $36 \%$ of patients with PSA value $<1 \mathrm{ng} / \mathrm{ml}$, $43 \%$ with PSA value between 1 and $2 \mathrm{ng} / \mathrm{ml}, 62 \%$ with PSA value between 2 and $3 \mathrm{ng} / \mathrm{ml}$ and $73 \%$ with a PSA value above $3 \mathrm{ng} / \mathrm{ml}$ [24].

An additional study of 170 patients with biochemical failure post-radical prostatectomy demonstrated that $44 \%$ of patients had a positive C11-choline PET scan. Interestingly, there appears to be an association between PSA doubling time (PSADT) and the ability of choline PET to detect recurrence. The percentage of patients with positive PET scans in whom the PSADT was $>6$ months was just $27 \%$, compared to $81 \%$ where the PSADT was $<3$ months [25]. Similarly, a correlation exists between PSA velocity (PSAV) and positive choline PET scans in patients with biochemical relapse after prostatectomy-patients with positive C11-choline PET scans have significantly higher PSAV $(p<0.05)$ than patients with negative scans [26]. These data suggest a relationship between PSA kinetics and choline uptake in the context of biochemical relapse. In summary, choline PET scanning is increasingly employed as a method for localising recurrent tumours in patients with biochemical relapse. Already, choline PET is being used to help plan whether with recurrent disease patients would benefit from localised or systemic therapies. A drawback of this technique is that it only appears to be useful once a diagnosis of biochemical relapse has been made and is currently not able to detect recurrent disease before a rise in PSA is noted. 


\section{Prostate-Specific Membrane Antigen PET (PSMA PET) - a Technique for Detecting Very Early Recurrence}

Although choline-based PET imaging is increasingly used for the detection of recurrent disease following curative therapy, several studies have reported concerns regarding sensitivity and specificity at low PSA levels [27-29]. Consequently, there is a need for a reliable diagnostic test in patients presenting with early biochemical failure. With this in mind, radionuclide imaging agents that target the cell surface receptor PSMA are receiving increasing attention $[30 \bullet \cdot, 31,32]$. PSMA is expressed in the prostate, salivary glands, small intestine and kidneys. However, it is significantly overexpressed in prostate cancer cells when compared to the surrounding normal tissue [33, 34]. PSMA is a trans-membrane receptor that internalises bound ligands [35]. It is the internalisation of the ligand-radionuclide complex that is thought to account for the high-quality images produced by this technique [36].

Ga-68 PSMA-targeted diagnostic imaging has many advantages over traditional PSA testing, not least the ability to detect anatomically the location of lymph node metastases and distant soft tissue spread. Historically, salvage radiation therapy has been delivered to the prostatic bed without knowledge of the site of residual disease. This new modality opens the possibility of salvage surgical or radiation therapy that can be targeted to the correct location [37]. Two groups have published papers in 2015 examining the effectiveness of Ga-68 PSMA imaging for detecting recurrent prostate cancer [30••, 32]. Firstly, Ashfar-Oromich et al. performed a retrospective analysis in 319 patients who underwent Ga-68 PSMA imaging [30••]. In 292 of these patients, progressive disease was suspected following prior conventional treatment (e.g., radiation therapy/surgery). Ga-68 identified positive lesions in $50 \%$ of men with a PSA $<0.2 \mathrm{ng} / \mathrm{ml}$, and as expected, the likelihood of a positive lesion correlated to PSA levels. From this cohort, tissue from 42 patients was isolated for further histological testing (biopsy/surgery) allowing comparison of images with gold standard histology. Lesion-based analysis from these 42 men gave sensitivity, specificity, negative predictive value and positive predictive values of $76,100,91$ and $100 \%$, respectively. Secondly, Dietlein et al. have published a comparison of two PSMA-labelled radionuclides (Ga-68 and F-18). In 14 men with biochemical relapse, both PSMAlabelled isotopes identified positive lesions in $10 \mathrm{pa}-$ tients [32]. This study was limited by only two patients having histological confirmation of their positive lesions but did demonstrate the ability of this technology to highlight sites of residual disease or metastasis. Further studies are required with histological verification of positive lesions before it is adopted into standard practice.

\section{Detecting Skeletal Metastases and Other Distant Disease}

As alluded to earlier, the skeleton is a frequent site of metastases in prostate cancer, and the development of bony disease is an indicator of poor prognosis. For many years, conventional wisdom has held that the presence of skeletal spread indicates terminal disease. However, recent interest has emerged in the possibility of identifying patients with a low burden of metastatic disease (so-called oligo-metastases), with the aim of offering potentially curative or disease-controlling therapy [38-42]. Clearly, the success of this philosophy is likely to depend on the ability to detect bone disease as early as possible. The involvement of the skeleton in prostate cancer is an important cause of morbidity and mortality [43]. Skeletal involvement in prostate cancer is typically difficult to assess using traditional imaging modalities, such a plain X-ray and CT, especially in the context of osteoblastic metastases [44]. Scintigraphy has long been a mainstay of assessing bone disease through the use of radiolabelled Tc99m-methylene diphosphate (MDP) [45]. MDP uptake by the skeleton is a function of blood supply, bone turnover and osteoblastic activity. Although this technique is sensitive at detecting osteogenic activity, there are limitations. MDP bone scan findings reflect changes in the bone rather than visualising the tumour. Disease regression can be difficult to discern due to residual tracer uptake in healing bone [46]. It is unlikely that conventional scintigraphy possesses the sensitivity and specificity to detect tiny skeletal deposits of prostate cancer. To this end, molecular imaging modalities offer great promise in the early detection of bone metastases.

\section{F18-Sodium Fluoride (NaF) PET Scanning for Imaging Skeletal Disease}

Interest in F18-sodium fluoride (NaF) PET scanning as a tool for imaging prostate cancer bone metastases has grown in recent years. Like radiolabelled phosphate analogues, $\mathrm{NaF}$ is incorporated into the hydroxyapatite lattice and collagen matrix of the skeleton. NaF has a higher affinity to bone than MDP and therefore allows earlier imaging time and improved image quality [47]. Indeed, several studies have indicated that NaF PET performs better than MDP for the assessment of osteoblastic metastases. One notable study compares planar bone scintigraphy, bone SPECT, NaF PET and NaF-PET/CT in patients with localised, high-risk or metastatic prostate cancer [48]. The sensitivity and specificity for detecting bone lesions were significantly higher for NaF PET/CT (100 and $100 \%$, respectively) than for planar scintigraphy (70 and $57 \%$ ), SPECT (92 and $82 \%$ ) or $\mathrm{NaF}$ PET alone (100 and $62 \%$ ). These results favour NaF PET/CT over conventional scintigraphy for the accurate detection of skeletal disease in prostate cancer.

Although NaF PET/CT is generally regarded as superior to conventional NDP scintigraphy, no prospective studies yet 
exist demonstrating any benefit in patient staging or management and it is unclear whether $\mathrm{NaF}$ will provide more meaningful information. Further clarity is needed to demonstrate a definitive advantage of $\mathrm{NaF}$ over conventional bone scanning techniques, which are cheaper and more widely available.

\section{FDG-PET to Monitor Treatment Response in Advanced Disease States}

Many prostate tumours possess low glycolytic activity, leading to the widely held notion that FDG-PET scanning has limited value in the assessment of prostate cancer. This is likely to be true in the case of organ-confined disease, where tumours may display low levels of glycolysis and benign hyperplastic or inflammatory pathologies may lead to increased FDG uptake, leading to false-positive diagnoses [49, 50]. However, FDG-PET may play a role in advanced disease states, where it has been used to assess response to hormone and/or chemotherapy in the context of metastatic disease [51].

\section{The Future-Probing Genetic Abnormalities in Prostate Cancer}

Molecular imaging techniques currently used in the clinical setting to assess prostate cancer largely employ the use of substrates taken up by metabolising tissues. This approach risks non-specificity for malignant cells, as well as missing tumours with low metabolic turnover. To this end, molecular probes are being developed that specifically target antigens selectively expressed by prostate cancer cells. In addition, cancer-specific probes are likely to be of great value as a tool to delineate the genetic heterogeneity of individual tumours and thus facilitate targeted, precision therapies $[8,9]$.

\section{Androgen Receptor (AR) Probes}

AR signalling is frequently implicated as a driver of tumour growth in prostate cancer, and anti-androgen therapy has long been a mainstay of the treatment of advanced disease. Invariably, after a period of response to hormone therapy, prostate tumours progress to a castrate-resistant state. This complex process is thought to involve either sensitisation or bypassing of the AR signalling pathway [52].

The distribution of sites where AR is overexpressed can be imaged using the PET agent $\left[{ }^{18} \mathrm{~F}\right]$-fluoro-5-alpha-dihydrotestosterone (18F-FDHT) [53, 54]. A recent study evaluated the associations between morphological CT patterns, glycolytic activity and AR expression on PET and found that the number of bone lesions on CT, FDG-PET and FDHT-PET as well as the intensity of FDHT uptake are significantly associated with overall survival [55•]. Of particular promise is the potential use of FDHT as a pharmacodynamic marker of drug targeting. One study reported that enzalutamide (an AR receptor antagonist)-induced FDHT uptake changes in metastatic lesions could be considered a surrogate marker for response of prostate cancer metastases with AR overexpression to ADT [56].

\section{Conclusions}

Prostate cancer is an important cause of morbidity and mortality affecting men worldwide. Herein, we have discussed several new developments in molecular imaging techniques that are likely to see increasing adoption over the next decade and that have good potential to revolutionise the management of men with prostate cancer.

In the future, molecular imaging techniques are likely to play a role in the management of prostate cancer at all stages of the disease, from the use of hyperpolarised MR in organ-confined disease, to the use of choline or PSMA tracers to detect early recurrence, right through to the detection of distant disease with $\mathrm{NaF}$ and FDG-PET. Additionally, imaging techniques using cancer-specific molecular probes (AR probes are an example) are currently being developed that will provide a non-invasive method for sub-typing tumours based on genetic or biochemical signatures. Such advances will help move us into the era of precision medicine, whereby therapies are tailored according to the specific characteristics of any given tumour.

\section{Compliance with Ethical Standards}

Conflict of Interest Aaron Leiblich, Daniel Stevens and Prasanna Sooriakumaran each declare no potential conflicts of interest.

Human and Animal Rights and Informed Consent This article does not contain any studies with human or animal subjects performed by any of the authors.

Open Access This article is distributed under the terms of the Creative Commons Attribution 4.0 International License (http:// creativecommons.org/licenses/by/4.0/), which permits unrestricted use, distribution, and reproduction in any medium, provided you give appropriate credit to the original author(s) and the source, provide a link to the Creative Commons license, and indicate if changes were made.

\section{References}

Papers of particular interest, published recently, have been highlighted as:

- Of importance

- Of major importance

1. Torre LA et al. Global cancer statistics, 2012. CA Cancer J Clin 2015;65(2):87-108. 
2. Siegel R et al. Cancer statistics, 2014. CA Cancer J Clin. 2014;64(1):9-29.

3. DeSantis CE et al. Cancer treatment and survivorship statistics, 2014. CA Cancer J Clin. 2014;64(4):252-71.

4. Brenner $\mathrm{H}$, Arndt $\mathrm{V}$. Long-term survival rates of patients with prostate cancer in the prostate-specific antigen screening era: population-based estimates for the year 2000 by period analysis. $\mathrm{J}$ Clin Oncol. 2005;23(3):441-7.

5. Sammon JD et al. Predicting life expectancy in men diagnosed with prostate cancer. Eur Urol. 2015;68(5):756-65.

6. Weissleder R, Mahmood U. Molecular imaging. Radiology. 2001;219(2):316-33.

7. Garraway LA, Lander ES. Lessons from the cancer genome. Cell. 2013;153(1):17-37.

8. Jackson SE, Chester JD. Personalised cancer medicine. Int J Cancer. 2015;137(2):262-6.

9. Kircher MF, Hricak H, Larson SM. Molecular imaging for personalized cancer care. Mol Oncol. 2012;6(2):182-95.

10. Penet MF et al. Molecular imaging of the tumor microenvironment for precision medicine and theranostics. Adv Cancer Res. 2014; 124:235-56.

11. Scheenen TW et al. Multiparametric magnetic resonance imaging in prostate cancer management: current status and future perspectives. Invest Radiol. 2015;50(9):594-600.

12. Futterer JJ et al. Can clinically significant prostate cancer be detected with multiparametric magnetic resonance imaging? A systematic review of the literature. Eur Urol. 2015;68(6):1045-53.

13. Kwee SA et al. Prostate cancer localization with 18 fluorine fluorocholine positron emission tomography. J Urol. 2005;173(1): 252-5.

14. Scher B et al. Value of $11 \mathrm{C}$-choline PET and PET/CT in patients with suspected prostate cancer. Eur J Nucl Med Mol Imaging. 2007;34(1):45-53.

15. Yamaguchi $\mathrm{T}$ et al. Prostate cancer: a comparative study of 11Ccholine PET and MR imaging combined with proton MR spectroscopy. Eur J Nucl Med Mol Imaging. 2005;32(7):742-8.

16. Nelson SJ et al. Metabolic imaging of patients with prostate cancer using hyperpolarized [1-(1)(3)C]pyruvate. Sci Transl Med. 2013;5(198):198ra108. This important paper describes the first-in-man study evaluating the safety and utility of hyperpolarised pyruvate MR in imaging prostate cancer. The authors demonstrate the safety of this technique and also its efficacy in detecting biopsy proven prostate cancer.

17. Wilson DM, Kurhanewicz J. Hyperpolarized 13C MR for molecular imaging of prostate cancer. J Nucl Med. 2014;55(10):1567-72.

18. Chen AP et al. Hyperpolarized C-13 spectroscopic imaging of the TRAMP mouse at 3T-initial experience. Magn Reson Med. 2007;58(6):1099-106.

19. Lupo JM et al. Analysis of hyperpolarized dynamic 13C lactate imaging in a transgenic mouse model of prostate cancer. Magn Reson Imaging. 2010;28(2):153-62.

20. Simmons MN, Stephenson AJ, Klein EA. Natural history of biochemical recurrence after radical prostatectomy: risk assessment for secondary therapy. Eur Urol. 2007;51(5):1175-84.

21. Morigi JJ et al. Prospective comparison of $18 \mathrm{~F}$-fluoromethylcholine versus $68 \mathrm{Ga}$-PSMA PET/CT in prostate cancer patients who have rising PSA after curative treatment and are being considered for targeted therapy. J Nucl Med. 2015;56(8):1185-90.

22. Souvatzoglou $\mathrm{M}$ et al. The sensitivity of [11C]choline PET/CT to localize prostate cancer depends on the tumor configuration. Clin Cancer Res. 2011;17(11):3751-9.

23. Punnen $\mathrm{S}$ et al. Management of biochemical recurrence after primary treatment of prostate cancer: a systematic review of the literature. Eur Urol. 2013;64(6):905-15.

24. Krause BJ et al. The detection rate of [11C]choline-PET/CT depends on the serum PSA-value in patients with biochemical recurrence of prostate cancer. Eur J Nucl Med Mol Imaging. 2008;35(1):18-23.

25. Giovacchini $\mathrm{G}$ et al. PSA doubling time for prediction of $[(11) \mathrm{C}]$ choline PET/CT findings in prostate cancer patients with biochemical failure after radical prostatectomy. Eur J Nucl Med Mol Imaging. 2010;37(6):1106-16.

26. Schillaci $\mathrm{O}$ et al. Influence of PSA, PSA velocity and PSA doubling time on contrast-enhanced $18 \mathrm{~F}$-choline PET/CT detection rate in patients with rising PSA after radical prostatectomy. Eur J Nucl Med Mol Imaging. 2012;39(4):589-96.

27. Heinisch $\mathrm{M}$ et al. Positron emission tomography/computed tomography with F-18-fluorocholine for restaging of prostate cancer patients: meaningful at PSA $<5 \mathrm{ng} / \mathrm{ml}$ ? Mol Imaging Biol. 2006;8(1):43-8.

28. Igerc I et al. The value of $18 \mathrm{~F}$-choline $\mathrm{PET} / \mathrm{CT}$ in patients with elevated PSA-level and negative prostate needle biopsy for localisation of prostate cancer. Eur J Nucl Med Mol Imaging. 2008;35(5):976-83.

29. Vees $\mathrm{H}$ et al. 18F-choline and/or 11C-acetate positron emission tomography: detection of residual or progressive subclinical disease at very low prostate-specific antigen values $(<1 \mathrm{ng} / \mathrm{mL})$ after radical prostatectomy. BJU Int. 2007;99(6):1415-20.

30.• Afshar-Oromieh A et al. The diagnostic value of PET/CT imaging with the (68)Ga-labelled PSMA ligand HBED-CC in the diagnosis of recurrent prostate cancer. Eur J Nucl Med Mol Imaging. 2015;42(2):197-209. This study evaluates the diagnostic value PSMA PET/CT as a tool for detecting recurrent prostate cancer in a large cohort of 319 patients. The authors demonstrate a high sensitivity for this technique as a tool for detecting recurrent disease. Of interest, recurrent lesions were even identified in men with PSA $<0.2 \mathrm{ng} / \mathrm{ml}$, demonstrating the utility of this approach in detecting very early recurrence.

31. Budaus L, et al. Initial experience of Ga-PSMA PET/CT imaging in high-risk prostate cancer patients prior to radical prostatectomy. Eur Urol. 2015.

32. Dietlein $\mathrm{M}$ et al. Comparison of [(18)F]DCFPyL and [(68)Ga]GaPSMA-HBED-CC for PSMA-PET imaging in patients with relapsed prostate cancer. Mol Imaging Biol. 2015;17(4):575-84.

33. Sweat SD et al. Prostate-specific membrane antigen expression is greatest in prostate adenocarcinoma and lymph node metastases. Urology. 1998;52(4):637-40.

34. Mannweiler S et al. Heterogeneity of prostate-specific membrane antigen (PSMA) expression in prostate carcinoma with distant metastasis. Pathol Oncol Res. 2009;15(2):167-72.

35. Rajasekaran SA et al. A novel cytoplasmic tail MXXXL motif mediates the internalization of prostate-specific membrane antigen. Mol Biol Cell. 2003;14(12):4835-45.

36. Eder $\mathrm{M}$ et al. PSMA as a target for radiolabelled small molecules. Eur J Nucl Med Mol Imaging. 2013;40(6):819-23.

37. Stenzl A. Salvage lymph node dissection in recurrent prostate cancer patients. Eur Urol. 2011;60(5):944-5.

38. Ahmed KA et al. Stereotactic body radiation therapy in the treatment of oligometastatic prostate cancer. Front Oncol. 2012;2:215.

39. Schick $U$ et al. Androgen deprivation and high-dose radiotherapy for oligometastatic prostate cancer patients with less than five regional and/or distant metastases. Acta Oncol. 2013;52(8):1622-8.

40. Decaestecker K et al. Repeated stereotactic body radiotherapy for oligometastatic prostate cancer recurrence. Radiat Oncol. 2014;9:135.

41. Bayne CE, et al. Treatment of the primary tumor in metastatic prostate cancer: current concepts and future perspectives. Eur Urol, 2015.

42. Sooriakumaran $\mathrm{P}$, et al. A multi-institutional analysis of perioperative outcomes in 106 men who underwent radical prostatectomy for distant metastatic prostate cancer at presentation. Eur Urol, 2015. 
43. Bubendorf $\mathrm{L}$ et al. Metastatic patterns of prostate cancer: an autopsy study of 1,589 patients. Hum Pathol. 2000;31(5):578-83.

44. Eisenhauer EA et al. New response evaluation criteria in solid tumours: revised RECIST guideline (version 1.1). Eur J Cancer. 2009;45(2):228-47.

45. Subramanian G, McAfee JG. A new complex of $99 \mathrm{mTc}$ for skeletal imaging. Radiology. 1971;99(1):192-6.

46. Pollen JJ, Witztum KF, Ashburn WL. The flare phenomenon on radionuclide bone scan in metastatic prostate cancer. AJR Am J Roentgenol. 1984;142(4):773-6.

47. Segall $\mathrm{G}$ et al. SNM practice guideline for sodium $18 \mathrm{~F}$-fluoride PET/CT bone scans 1.0. J Nucl Med. 2010;51(11):1813-20.

48. Even-Sapir E et al. The detection of bone metastases in patients with high-risk prostate cancer: $99 \mathrm{mTc}-\mathrm{MDP}$ Planar bone scintigraphy, single- and multi-field-of-view SPECT, 18F-fluoride PET, and 18F-fluoride PET/CT. J Nucl Med. 2006;47(2):287-97.

49. Hillner BE et al. Relationship between cancer type and impact of PET and PET/CT on intended management: findings of the national oncologic PET registry. J Nucl Med. 2008;49(12):1928-35.

50. Hillner BE et al. Impact of positron emission tomography/ computed tomography and positron emission tomography (PET) alone on expected management of patients with cancer: initial results from the National Oncologic PET Registry. J Clin Oncol. 2008;26(13):2155-61.
51. Morris MJ et al. Fluorodeoxyglucose positron emission tomography as an outcome measure for castrate metastatic prostate cancer treated with antimicrotubule chemotherapy. Clin Cancer Res. 2005;11(9):3210-6.

52. Karantanos $\mathrm{T}$ et al. Understanding the mechanisms of androgen deprivation resistance in prostate cancer at the molecular level. Eur Urol. 2015;67(3):470-9.

53. Liu A et al. Fluorine-18-labeled androgens: radiochemical synthesis and tissue distribution studies on six fluorine-substituted androgens, potential imaging agents for prostatic cancer. J Nucl Med. 1992;33(5):724-34.

54. Larson SM et al. Tumor localization of 16beta-18F-fluoro5alpha-dihydrotestosterone versus $18 \mathrm{~F}-\mathrm{FDG}$ in patients with progressive, metastatic prostate cancer. J Nucl Med. 2004;45(3): 366-73.

55. Vargas HA et al. Bone metastases in castration-resistant prostate cancer: associations between morphologic CT patterns, glycolytic activity, and androgen receptor expression on PET and overall survival. Radiology. 2014;271(1):220-9. his study demonstrates that in men with castrate-resistant disease the number of bone lesions on FDHT PET/CT and the intensity of FDHT uptake is significantly associated with survival.

56. Scher HI et al. Antitumour activity of MDV3100 in castrationresistant prostate cancer: a phase 1-2 study. Lancet. 2010;375(9724):1437-46. 